\section{Gastos do Sistema Único de Saúde com internações por causas externas em São José dos Campos, São Paulo, Brasil}

\author{
Unified National Health System costs in \\ São José dos Campos, São Paulo State, Brazil, \\ for hospital admissions due to external causes
}

\footnotetext{
1 Secretaria Municipal de Saúde, Prefeitura Municipal de São José dos Campos, São José dos Campos, Brasil. 2 Faculdade de Saúde Pública, Universidade de São Paulo, São Paulo, Brasil.

Correspondência L. P. R. Melione Secretaria Municipal de Saúde, Prefeitura Municipal de São José dos Campos. Rua José de Alencar 123, $5^{\circ}$ andar, sala 1, São José dos Campos, SP 12209-530, Brasil.

lmelione@uol.com.br
}

\begin{abstract}
The objective of this study was to identify direct expenditures for hospitalizations due to external causes in the Unified National Health System (SUS) in the city of São José dos Campos, São Paulo State, Brazil. Admissions to the Dr. José de Carvalho Florence Municipal Hospital resulting from external causes or injuries - ICD-10, chapters XIX and XX respectively - were analyzed for the first semester of 2003. 976 patient admission forms were analyzed, after data evaluation. Admissions with the highest total cost were those resulting from motor vehicle accidents and falls. The highest mean cost for hospitalization for injury was due to motor vehicle accidents (BRL 614.63), followed by assault (BRL 594.90). The highest mean cost for hospitalization due to injury was for cervical fractures (BRL 1,191.42) and head injuries (BRL 1,000.44). Hospitalizations with the highest daily cost were skull and facial fractures (BRL 166.72) and abdominal trauma (BRL 148.26). The study confirmed that motor vehicle accidents, falls, and assault are an important source of costs due to hospitalization for injuries.
\end{abstract}

Health Expenditures; External Causes; Hospitalization; Single Health System
Luís Paulo Rodrigues Melione 1

Maria Helena Prado de Mello-Jorge 2

\section{Introdução}

O aumento dos acidentes e da violência (causas externas), no Brasil, tem repercutido na organização do sistema de saúde, o qual, por sua responsabilidade na atenção ao trauma, vem tendo seus gastos elevados com a assistência médica. Tanto no Brasil como no Estado de São Paulo, as causas externas correspondem a maior gasto médio e custo-dia de internação do que as causas naturais, apesar da menor proporção de internações e menor tempo médio de permanência daquelas 1. No Brasil, a proporção de internações por causas externas aumentou progressivamente, de 5,2\%, em 1998, para 6,9\%, em 2005, assim como a proporção de gastos, que passou de $6,4 \%$ para $8,5 \% 2$.

As quedas e os acidentes de transporte têm sido referidos, respectivamente, como a primeira e a segunda causas de internações por causas externas. São poucos os estudos sobre o impacto econômico dos acidentes e violências nos gastos governamentais da saúde ${ }^{3}$. No período de 1984 a 2003, foram localizados 18 estudos sobre custos da assistência médica hospitalar em artigo de revisão 4 . A maioria dos estudos realizados no Brasil e em outros países sobre o tema é específica, mensurando, por exemplo, os custos da assistência com quedas em idosos 5 , com acidentes de trânsito 6 e com traumas na infância 7, entre outros. 
Para Hodgson \& Meiners (1982, apud Iunes 8), o custo econômico de uma doença, ou problema de saúde, pode ser classificado em custos diretos e indiretos. Os custos diretos referem-se aos custos médicos (exames, procedimentos, consultas, internações, reabilitação e outros) e aos custos não médicos, às despesas de parentes e acompanhantes com transporte e dietas especiais, por exemplo. Os custos indiretos são os relacionados com a perda de produção e produtividade decorrentes da doença ou problema de saúde.

No Brasil, o Sistema de Informações Hospitalares do Sistema Único de Saúde (SIH-SUS) permite a captação de dados referentes aos valores pagos pelo Ministério da Saúde aos hospitais, quantias que representam parte dos custos diretos médico-hospitalares. Essas somas baseiam-se em uma tabela de valores de procedimentos hospitalares ${ }^{9}$, que atribui valores médios por componentes de custo de internação com diferentes pesos. São, principalmente, serviços hospitalares, serviços profissionais, serviços auxiliares diagnóstico-terapêuticos, materiais e medicamentos, órteses e próteses, UTI e sangue. Ocorre que muitos dos valores constantes dessa tabela estão defasados em relação aos valores reais efetivamente despendidos pelos hospitais, e a diferença entre essas quantias é assumida com recursos próprios pelos respectivos serviços.

De acordo com Iunes 8 (p. 40), “...os valores absolutos e os pesos relativos dos diversos componentes da AIH estão muito mais relacionados com limitações orçamentárias do que com a estrutura de custos do procedimento". Por esta razão, diz-se que os valores pagos pelo Ministério da Saúde não refletem os custos diretos verdadeiros da assistência hospitalar pública e, sim, os gastos do Governo Federal com o problema. No entanto, a inexistência de outra fonte de informação disponível com valores mais próximos do custo real torna a base de dados do SIH-SUS uma fonte importante de dados para análise dos gastos com internação hospitalar pelo SUS, desde que se leve em consideração essa limitação.

Embora as internações pagas pelo SUS não representem a totalidade dos casos que necessitam internação hospitalar, o caráter de urgência dos atendimentos às lesões provocadas por causas externas conferem grande cobertura de assistência ao SIH-SUS nesse grupo de problemas de saúde.

Até 1997, os dados de causas externas no SIHSUS referiam-se apenas à natureza da lesão que levou à internação. Somente a partir de 1998, o Ministério da Saúde implantou no SIH-SUS, por meio da Portaria $n^{o} .142$ 10, de 13 de novembro de 1997, a obrigatoriedade do registro, no campo "diagnóstico principal", do código da Clas- sificação Internacional de Doenças, $10^{\mathrm{a}}$ revisão (CID-10) 11 referente à natureza da lesão (capítulo XIX) e, no campo "diagnóstico secundário", do código referente à causa externa (capítulo $\mathrm{XX}$ ). Esse marco legal subsidiou a implantação de uma base nacional de dados sobre morbidade hospitalar por causas externas, com finalidade epidemiológica, além de administrativa.

O objetivo deste estudo foi mensurar os gastos diretos médico-hospitalares das internações por causas externas em um hospital público de São José dos Campos, Estado de São Paulo, Brasil, que é referência para o atendimento ao trauma no município.

\section{Material e métodos}

O Município de São José dos Campos localiza-se a leste do Estado de São Paulo, no Vale do Paraíba. Está localizado entre as duas principais regiões metropolitanas do país e vem se consolidando como um pólo industrial de grande porte e também como um centro comercial e prestador de serviços para a região 12. Em 2000, sua população chegou a 539.313 habitantes (Instituto Brasileiro de Geografia e Estatística. Censo demográfico de 2000. http://www.ibge.gov.br). Apresenta riscos para a ocorrência de acidentes e violências em virtude da sua extensa e movimentada malha viária urbana e por abrigar, em seu território, parte de quatro importantes eixos rodoviários da região: BR 116 - Rodovia Presidente Dutra; SP 70 - Rodovia Carvalho Pinto; SP 99 - Rodovia dos Tamoios; SP 50 - Estrada Velha de Campos do Jordão.

A rede de assistência médica de emergência do município era formada, à época do estudo, pelo hospital municipal (referência formal e com maior resolutividade para o atendimento ao trauma), por três unidades de pronto-atendimento (atendimento clínico e para casos menos graves) e por três hospitais filantrópicos conveniados ao SUS (internações clínicas e cirúrgicas).

Foram estudadas as internações por causas externas pagas pelo SUS no Hospital Municipal Dr. José de Carvalho Florence, em São José dos Campos, entre $1^{\circ}$ de janeiro e 30 de junho de 2003. Nesse hospital, concentraram-se $92,3 \%$ das internações registradas como lesões decorrentes de causas externas pelo SUS no mesmo período. As internações nos demais hospitais, se agregadas às do hospital municipal numa avaliação preliminar dos indicadores administrativos do SIHSUS do município, alterariam o gasto médio de internação, de $\mathrm{R} \$ 488,57$ para $\mathrm{R} \$ 528,61$; o custodia, de $\mathrm{R} \$ 74,97$ para $\mathrm{R} \$$ 83,52; o tempo médio de permanência, de 6,5 para 6,3 dias. A proporção 
de gastos de internação por tipo de causa externa também seria alterada: no caso das internações por acidentes de transporte, de $38 \%$ para $32,8 \%$; nas quedas, de $23,3 \%$ para $22,8 \%$; nas causas indeterminadas, de $18 \%$ para $15,9 \%$; nas agressões, de $9,6 \%$ para $8,5 \%$; nas demais causas acidentais, de $6,4 \%$ para $5,5 \%$; nas complicações de procedimentos médicos, de $4,2 \%$ para $9,8 \%$; nas seqüelas de causas externas, de 3,9\% para 3,4\% 2 .

Sabe-se que a cobertura de internações por todas as doenças do setor público, em relação às internações financiadas por particulares e convênios médicos, na região de São José dos Campos, em 2002, foi de $81 \% 13$.

Foram selecionadas todas as internações (de residentes e não residentes no município) cujo diagnóstico principal estivesse contido, independentemente, no capítulo XIX (lesões, envenenamentos e algumas outras conseqüências de causas externas) da CID-10 11 ou no capítulo XX (causas externas de morbidade e de mortalidade); cujo diagnóstico secundário constasse do capítulo XIX ou XX. Com esse procedimento, procurou-se recuperar registros que, eventualmente, tivessem sido codificados em desacordo com as regras de morbidade da CID-10 11 e do próprio SIH-SUS 10.

Foram selecionadas as Autorizações de Internação Hospitalar (AIHs) do tipo 1, de modo a não incluir as internações de longa permanência. Levou-se em consideração o fato de que as 27 internações de longa permanência excluídas do estudo eram por seqüelas de causas externas, pouco influindo nos resultados das tabulações pelos demais tipos de causas externas, mas influindo nos resultados gerais pelos seus extremos distantes da média. As AIHs de longa permanência merecem análise em separado, pois apresentam gasto médio elevado, custo-dia baixo e tempo médio de permanência longo.

Os procedimentos metodológicos adotados neste estudo seguiram o modelo da pesquisa original da qual seus dados foram extraídos, em que foram avaliadas: (a) a qualidade dos dados registrados no banco de dados do SIH-SUS em comparação com aqueles registrados no prontuário do paciente; (b) a morbidade hospitalar a partir dos dados do SIH-SUS corrigidos após consulta aos prontuários; (c) os gastos de internação corrigidos após consulta aos prontuários 14 .

\section{Fontes de dados}

Base de dados de AIHs pagas pelo Ministério da Saúde. Essa base de dados foi obtida dos arquivos públicos disponíveis em meio eletrônico, na página do Departamento de Informática do SUS (DATASUS) 2, na internet. As bases de dados do ano de competência 2003 (janeiro a dezembro) foram consultadas para a seleção das internações com data de entrada entre $1^{\circ}$ de janeiro e 30 de junho de 2003.

Base de dados de AIHs identificadas e apresentadas pelo município. Os dados foram obtidos do programa SISAIH01 - sistema de AIH em disquete, desenvolvido pelo DATASUS (DATASUS, Brasília, Brasil), disponível no município. Os critérios para consultas às bases de dados, para seleção dos diagnósticos principal e secundário e do tipo de AIH foram os mesmos citados no item anterior

Prontuários médicos dos pacientes internados por causas externas. Os números dos prontuários foram obtidos do banco de dados de AIHs identificadas do município. Esses documentos estavam no hospital municipal, tendo sido encontrados 990 dos 993 prontuários de pacientes internados por causas externas por esse método.

O processo de preparo das bases de dados para a pesquisa iniciou-se com a procura de internações por lesões provocadas por causas externas nos arquivos de dados de AIHs pagas, as quais foram localizadas e selecionadas pelos critérios já informados. Posteriormente, foi realizada a mesma busca para as AIHs identificadas. Assim, foram vinculadas todas as internações pagas, em banco de dados não identificados, com aquelas AIHs identificadas apresentadas pelo município.

As bases de dados de AIHs pagas e AIHs identificadas foram relacionadas e transformadas em um único banco de dados, com o número do prontuário da paciente. A chave primária de relacionamento desses arquivos de dados de internação hospitalar foi o número da AIH, o mês e o ano de competência. Dessa forma, as AIHs reapresentadas no período foram consideradas, no lugar daquelas recusadas quando da sua primeira apresentação.

As variáveis incluídas no estudo foram: Diagnóstico Principal, Diagnóstico Secundário e Valor Pago.

\section{Diagnóstico principal}

Definido como a afecção primariamente responsável pela necessidade de tratamento ou investigação do paciente. Foram selecionados os diagnósticos principais de internação, com os códigos de categorias de três caracteres da CID-10 11: S00 a T98, que representam as "lesões, envenenamentos e algumas outras conseqüências de causas externas" (capítulo XIX), sendo constituídos dois grupos de análise, um com ênfase no segmento corporal afetado e outro, na natureza da lesão. 
De acordo com o segmento corporal afetado: S00-S09 (cabeça), S10-S19 (pescoço), S20-S29 (tórax), S30-S39 (abdome, dorso e pelve), S40S69 (membros superiores), S70-S99 (membros inferiores) e T00-T07 (traumatismos múltiplos). Os agrupamentos para os quais não foi possível indicar um segmento corporal afetado foram mantidos como na classificação de natureza da lesão.

Com relação à natureza da lesão: S02 (fratura de crânio e ossos da face), S12 (fratura do pescoço), S22 (fratura do tórax: costelas, esterno e coluna torácica), S32 (fratura de coluna lombar e da pelve), S42 (fratura do ombro e do braço), S52 (fratura do antebraço), S62 (fratura do punho e da mão), S72 (fratura do fêmur), S82 (fratura da perna, incluindo tornozelo) e S92 (fratura do pé); S03, S13, S23, S33, S43, S53, S63, S73, S83, S93 e T03 (luxações, entorses e distensões), S06 (traumatismo intracraniano), S27 (traumatismo de outros órgãos intratorácicos), S36 (traumatismo de órgãos intra-abdominais), T01-T07 (traumatismos múltiplos), T20 a T32 (queimaduras e corrosões), T36 a T65 (intoxicações), T79-T88 (complicações de cuidados médicos e cirúrgicos), T90-T98 (seqüelas de causas externas); todas as demais categorias do capítulo XIX (demais lesões).

\section{Diagnóstico secundário}

O diagnóstico secundário foi obrigatoriamente uma causa externa, desde que o diagnóstico principal tivesse sido uma lesão, segundo regra internacional de codificação da CID-10 11 e do SIH-SUS 10. Foram selecionados os diagnósticos secundários de internação, com os códigos de categorias V01-Y98, que representam as causas externas de morbidade e de mortalidade da CID-10 (capítulo XX). Constituíram-se de agrupamentos, segundo as categorias: V01-V99 (acidentes de transporte), W01-W19 (quedas), X85-Y09 e Y35-Y36 (agressões), X60-X84 (lesões autoprovocadas intencionalmente), Y40-Y84 (complicações de assistência médica e cirúrgica), Y10-Y34 (eventos cuja intenção é indeterminada), Y85Y89 (seqüelas de causas externas); todas as demais categorias do capítulo XX (demais causas acidentais).

\section{Valor pago}

Correspondendo ao valor, em reais, pago pelo Ministério da Saúde pelas internações. O gasto médio de internação, o custo-dia e o tempo médio de permanência foram calculados pelas fórmulas a seguir: Gasto médio de internação $=\frac{\text { Valor pago pelas internações }}{\text { Número de internações }}$

Custo-dia $=\frac{\text { Valor pago pelas internações }}{\text { Número de dias de permanência }}$

Tempo médio de permanência $=\frac{\text { Número de dias de permanência }}{\text { Número de internaç̃̃es }}$

O tipo de saída hospitalar foi categorizado em "alta" e "óbito".

Para as tabulações de número, proporção, razão e valores pagos pelas internações, oriundos do SIH-SUS, e dos dados originais coletados de prontuários, foi utilizado o programa de domínio público Epi Info (Centers for Disease Control and Prevention, Atlanta, Estados Unidos). A população de estudo, para a análise dos indicadores valor total, tempo médio de permanência, gasto médio e custo-dia, foi de 976 internações, que correspondeu ao número de internações por lesões e causas externas confirmadas após avaliação dos prontuários dos pacientes.

A pesquisa envolveu a consulta às bases de dados de AIHs identificadas e aos prontuários médicos dos pacientes. O projeto de pesquisa foi aprovado pelo Comitê de Ética e Pesquisa da Faculdade de Saúde Pública da Universidade de São Paulo em 23 de março de 2005, protocolo de pesquisa $\mathrm{n}^{\circ}$. 1.230.

\section{Resultados}

Foram encontrados 990 dos 993 prontuários de pacientes internados por causas externas $(99,7 \%)$. Destas, cinco AIHs codificadas como causas externas no SIH-SUS foram descaracterizadas como tal na avaliação do pesquisador, e em nove casos não havia informação suficiente para concluir se a internação era decorrente de causa externa ou causa natural, já que o procedimento e a lesão eram compatíveis com as duas possibilidades. A população de estudo foi, portanto, de 976 internações, sem exclusão de reinternações. A proporção de reinternações foi de 10,6\%; das 976 internações, 73,5\% ocorreram na seqüência do agravo, caracterizando o hospital como porta de entrada para o sistema de atendimento de emergência do município.

No presente estudo, as lesões segundo segmento corporal afetado que apresentaram maior gasto médio por internação foram, respectivamente: pescoço ( $\mathrm{R} \$ 980,26)$, cabeça ( $\mathrm{R} \$ 684,83)$, traumatismos múltiplos (R\$671,26) e traumatismos de abdome (R\$ 654,45) (Tabela 1). Em São José dos Campos, apesar de o trauma de cabeça ter menor gasto médio que o trauma de pescoço, o seu custo-dia foi quase o dobro, em virtude do maior tempo médio de permanência $(17,1$ 
Internações por causas externas, tempo médio de permanência, custo-dia, gasto médio, segundo segmento corporal afetado. Hospital Municipal de São José dos Campos, São José dos Campos, São Paulo, Brasil, primeiro semestre de 2003.

\begin{tabular}{lcccc}
\hline Segmento corporal afetado & $\mathbf{n}$ & $\begin{array}{c}\text { Tempo médio de } \\
\text { permanência (dias) }\end{array}$ & $\begin{array}{c}\text { Custo-dia } \\
\text { (em Reais) }\end{array}$ & $\begin{array}{c}\text { Gasto médio } \\
\text { (em Reais) }\end{array}$ \\
\hline Cabeça & 202 & 6,1 & 112,93 & 684,83 \\
Pescoço & 14 & 17,1 & 57,18 & 980,26 \\
Tórax & 29 & 7,2 & 79,23 & 571,03 \\
Abdome & 100 & 7,6 & 85,77 & 654,45 \\
Membros superiores & 211 & 4,3 & 54,15 & 231,99 \\
Membros inferiores & 258 & 8,2 & 55,08 & 453,01 \\
Traumatismos múltiplos & 50 & 7,3 & 92,46 & 671,26 \\
Queimaduras & 11 & 3,8 & 130,18 & 497,06 \\
Intoxicações & 25 & 4,4 & 106,67 & 473,60 \\
Complicações de cuidados médicos & 59 & 7,4 & 45,13 & 334,27 \\
Seqüelas & 10 & 7,8 & 64,27 & 501,33 \\
Demais lesões & 7 & 3,9 & 74,94 & 289,07 \\
Todas as lesões & 976 & 6,7 & 73,23 & 489,26 \\
\hline
\end{tabular}

dias) desse último tipo de trauma, que demanda imobilização prolongada.

Na Tabela 2, observa-se que a natureza da lesão responsável pelo maior gasto médio foi fratura de pescoço ( $R \$ 1.191,42)$, seguida pelo traumatismo intracraniano $(\mathrm{R} \$ 1.000,44)$ e traumatismo intra-abdominal ( $\mathrm{R} \$ \mathbf{8 1 3}, 78)$. As queimaduras corresponderam ao maior custo-dia (R\$130,18), embora representassem o menor número de ocorrências. Isso se deveu ao tempo médio de permanência curto, de 3,8 dias. Como este não é um hospital especializado em tratamento de queimados, os casos mais graves são transferidos para centros especializados.

Pode-se perceber, pelos números e valores da Tabela 3, que os acidentes de transporte foram não só a principal causa de internação $(32,8 \%)$, como também a primeira causa nos gastos $(41,2 \%)$. As quedas ficaram em segundo lugar na freqüência de internações $(26,6 \%)$ e nos gastos $(25,7 \%)$. Chamou a atenção o terceiro lugar para as causas indeterminadas: $18,9 \%$ para freqüência e $15,2 \%$ para os gastos.

$\mathrm{Na}$ análise da distribuição de gastos e permanência por tipo de causa externa (Tabela 3), pode-se perceber que os acidentes de transporte foram responsáveis pelo maior gasto médio de internação (R\$ 614,63). As agressões apresentaram, mesmo com número menor de internações, o segundo maior gasto médio ( $\mathrm{R} \$ 594,90)$ e o maior custo-dia, atrelado ao menor tempo médio de permanência para as lesões decorrentes desse tipo de agravo, que foram as fraturas de face e o traumatismo intracraniano.
Conforme referido, as fraturas de pescoço, o traumatismo intracraniano e o traumatismo intra-abdominal tiveram os maiores gastos médios de internação por causas externas. No caso da fratura do pescoço, seu gasto médio foi maior nos acidentes de transporte ( $\mathrm{R} \$ 1.213,85)$ do que nas quedas ( $\mathrm{R} \$ 1.003,10)$. O gasto médio de traumatismo intracraniano nas agressões foi de $\mathrm{R} \$$ 1.179,99; nos acidentes de transporte foi de R\$ $1.113,19$; nas quedas, de $\mathrm{R} \$ 816,87$; nas demais causas acidentais foi de $\mathrm{R} \$ 767,94$. No traumatismo intra-abdominal, o gasto médio foi maior nas agressões (R\$ 877,71), nas demais causas acidentais $(\mathrm{R} \$ 770,90)$ e nos acidentes de transporte $(\mathrm{R} \$$ 755,69) (Tabela 4).

A análise da Tabela 4 permitiu identificar um elevado gasto médio de internação por causas indeterminadas nas fraturas de pescoço (R\$ $1.644,20)$, intoxicações ( $\mathrm{R} \$ 1.106,96)$, traumatismo intracraniano ( $\mathrm{R} \$ 1.064,77)$ e traumatismo intra-abdominal (R\$ 909,61). Traumas relevantes deixaram de ser classificados dentro do tipo de causa externa correto por falta de informação detalhada sobre a causa externa nos prontuários dos pacientes.

Os casos que evoluíram para óbito tiveram maior gasto médio de internação do que os casos que tiveram alta em quaisquer que fossem as lesões ou causas externas envolvidas (Tabela 5). Optou-se pela utilização de uma "razão de gasto médio óbito/alta”, obtida pela divisão do gasto médio de saídas hospitalares por óbito, pelo gasto médio das saídas por alta. Esse método é mais adequado para comparação com outros 
Internações por causas externas, tempo médio de permanência, custo-dia e gasto médio, segundo natureza da lesão. Hospital Municipal de São José dos Campos, São José dos Campos, São Paulo, Brasil, primeiro semestre de 2003.

\begin{tabular}{|c|c|c|c|c|}
\hline Natureza da lesão & $\mathbf{n}$ & $\begin{array}{l}\text { Tempo médio de } \\
\text { permanência (dias) }\end{array}$ & $\begin{array}{l}\text { Custo-dia } \\
\text { (em Reais) }\end{array}$ & $\begin{array}{c}\text { Gasto médio } \\
\text { (em Reais) }\end{array}$ \\
\hline Fraturas & 511 & 7,1 & 56,21 & 401,72 \\
\hline Crânio e ossos da face & 64 & 1,3 & 166,72 & 224,03 \\
\hline Pescoço & 9 & 18,3 & 64,99 & $1.191,42$ \\
\hline Tórax & 9 & 12,1 & 49,98 & 605,31 \\
\hline Coluna lombar e pelve & 37 & 12,2 & 53,24 & 648,97 \\
\hline Ombro e braço & 35 & 14,3 & 18,61 & 265,31 \\
\hline Antebraço & 81 & 2,3 & 117,06 & 273,15 \\
\hline Punho e mão & 39 & 3,2 & 52,54 & 167,06 \\
\hline Fêmur & 115 & 12,8 & 48,13 & 617,35 \\
\hline Perna & 103 & 4,8 & 76,99 & 368,49 \\
\hline Pé & 19 & 3,2 & 63,68 & 204,45 \\
\hline Luxações, entorses e distensões & 32 & 5,0 & 55,41 & 277,05 \\
\hline Traumatismo intracraniano & 122 & 9,2 & 109,17 & $1.000,44$ \\
\hline Traumatismo do tórax & 15 & 4,5 & 98,20 & 445,18 \\
\hline Traumatismo intra-abdominal & 45 & 5,5 & 148,26 & 813,78 \\
\hline Traumatismos múltiplos & 50 & 7,3 & 92,46 & 671,26 \\
\hline Queimaduras & 11 & 3,8 & 130,18 & 497,06 \\
\hline Intoxicações & 25 & 4,4 & 106,67 & 473,60 \\
\hline Complicações de cuidados médicos & 59 & 7,4 & 45,13 & 334,27 \\
\hline Seqüelas & 10 & 7,8 & 64,27 & 501,33 \\
\hline Demais lesões & 96 & 2,6 & 91,50 & 233,52 \\
\hline Todas as lesões & 976 & 6,7 & 73,23 & 489,26 \\
\hline
\end{tabular}

estudos de bases de dados mais amplas, pois diferentes graus de complexidade da assistência hospitalar tornariam os valores de gasto médio pouco comparáveis. As lesões que tiveram maior razão de gasto médio óbito/alta foram: traumatismos múltiplos $(5,3: 1)$ e os traumatismos do tórax $(4,3: 1)$. As causas externas com maior razão gasto médio óbito/alta foram as causas indeterminadas $(4,1: 1)$, as quedas $(2,7: 1)$ e os acidentes de transporte $(2,6: 1)$ e agressões $(1,2: 1)$.

\section{Discussão}

Algumas limitações devem, inicialmente, ser ressaltadas neste estudo. A primeira se refere ao fato de que as internações hospitalares não retratam toda a morbidade por causas externas. Uma parcela importante das vítimas sequer é atendida em serviços de urgência/emergência e outra parcela significativa é atendida e liberada após o atendimento. As internações refletem, assim, provavelmente, a morbidade mais grave. A segunda diz respeito à fonte de financiamento das interna- ções, neste caso o SUS, o que exclui, portanto, as internações financiadas por particulares e por seguros de saúde. Contudo, as internações pelo SUS por causas externas representam a grande maioria das internações nesse grupo de agravos. A terceira limitação importante da pesquisa foi a restrição da análise dos dados a somente um hospital do município, embora 92,3\% das internações por lesões decorrentes de causas externas tivessem ocorrido nesse hospital, no período de estudo.

Uma análise com base nos dados de procedimentos realizados ou por unidades de custo de internação, disponíveis no banco de dados do SIH-SUS, seria relevante para esclarecer mais detalhes dos gastos de internações por causas externas. No entanto, este estudo não avaliou esses aspectos, pois, conforme mencionado anteriormente, foi parte de uma pesquisa que avaliou a qualidade dos dados de internação, e os procedimentos realizados não foram objeto de avaliação.

Sabe-se que os gastos do Ministério da Saúde com internações pelo SUS por causas externas 
Tabela 3

Internações por causas externas, valor total, tempo médio de permanência, gasto médio e custo-dia, segundo tipo de causa. Hospital Municipal de São José dos Campos, São José dos Campos, São Paulo, Brasil, primeiro semestre de 2003.

\begin{tabular}{|c|c|c|c|c|c|c|c|}
\hline \multirow[t]{2}{*}{ Tipo de causa } & \multicolumn{2}{|c|}{ Internações } & \multicolumn{2}{|c|}{ Valor total } & \multirow{2}{*}{$\begin{array}{l}\text { Tempo médio } \\
\text { de permanência } \\
\text { (dias) }\end{array}$} & \multirow{2}{*}{$\begin{array}{c}\text { Gasto } \\
\text { médio } \\
\text { (em Reais) }\end{array}$} & \multirow{2}{*}{$\begin{array}{l}\text { Custo-dia } \\
\text { (em Reais) }\end{array}$} \\
\hline & $\mathrm{n}$ & $\%$ & Reais & $\%$ & & & \\
\hline Acidentes de transporte & 320 & 32,8 & $196.682,76$ & 41,2 & 7,7 & 614,63 & 80,21 \\
\hline Quedas & 260 & 26,6 & $122.845,60$ & 25,7 & 8,7 & 472,48 & 54,28 \\
\hline Demais causas acidentais & 81 & 8,3 & $25.576,40$ & 5,4 & 3,2 & 315,76 & 99,52 \\
\hline Agressões & 49 & 5,0 & $29.149,89$ & 6,1 & 5,3 & 594,90 & 112,11 \\
\hline Lesões autoprovocadas intencionalmente & 12 & 1,2 & $6.007,34$ & 1,3 & 4,9 & 500,61 & 101,82 \\
\hline Complicações da assistência médica & 60 & 6,1 & $19.821,28$ & 4,2 & 7,4 & 330,35 & 44,84 \\
\hline Causas indeterminadas & 184 & 18,9 & $72.424,89$ & 15,2 & 3,9 & 393,61 & 102,01 \\
\hline Seqüelas & 10 & 1,0 & $5.013,31$ & 1,0 & 7,8 & 501,33 & 64,27 \\
\hline Todas as causas externas & 976 & 100,0 & $477.521,47$ & 100,0 & 6,7 & 489,26 & 73,23 \\
\hline
\end{tabular}

Tabela 4

Gasto médio de internação (em Reais), segundo tipo de causa externa e natureza da lesão. Hospital Municipal de São José dos Campos, São José dos Campos, São Paulo, Brasil, primeiro semestre de 2003 ( $N=976)$.

\begin{tabular}{|c|c|c|c|c|c|c|c|c|c|}
\hline Natureza da lesão & $\begin{array}{c}\text { Acidentes } \\
\text { de } \\
\text { transporte }\end{array}$ & Quedas & $\begin{array}{c}\text { Demais } \\
\text { causas } \\
\text { acidentais }\end{array}$ & Agressões & $\begin{array}{l}\text { Lesões } \\
\text { autopro- } \\
\text { vocadas }\end{array}$ & $\begin{array}{l}\text { Compli- } \\
\text { cações da } \\
\text { assistência } \\
\text { médica }\end{array}$ & $\begin{array}{l}\text { Causas } \\
\text { indeter- } \\
\text { minadas }\end{array}$ & Seqüelas & $\begin{array}{c}\text { Todos os } \\
\text { tipos de } \\
\text { causas } \\
\text { externas }\end{array}$ \\
\hline Fraturas & 466,25 & 410,32 & 379,15 & 194,77 & - & - & 309,07 & - & 401,72 \\
\hline Crânio e ossos da face & 296,03 & 185,03 & 181,34 & 171,85 & - & - & 195,80 & - & 224,03 \\
\hline Pescoço & $1.213,85$ & $1.003,10$ & - & - & - & - & $1.644,20$ & - & $1.191,42$ \\
\hline Tórax & 674,45 & 678,37 & - & 40,38 & - & - & - & - & 605,31 \\
\hline Coluna lombar e pelve & 578,23 & 711,33 & 873,94 & - & - & - & - & - & 648,97 \\
\hline Ombro e braço & 259,80 & 275,90 & - & 40,38 & - & - & 277,27 & - & 265,31 \\
\hline Antebraço & 250,76 & 284,26 & - & 311,42 & - & - & 278,38 & - & 273,15 \\
\hline Punho e mão & 98,98 & 170,56 & 208,94 & 40,38 & - & - & 188,88 & - & 167,06 \\
\hline Fêmur & 674,02 & 559,01 & - & 839,16 & - & - & 773,21 & - & 617,35 \\
\hline Perna & 443,60 & 294,37 & 394,25 & - & - & - & 330,79 & - & 368,49 \\
\hline Pé & 192,85 & 233,00 & 268,42 & - & - & - & 148,79 & - & 204,45 \\
\hline Luxações, entorses e distensões & 210,04 & 371,82 & - & - & - & - & 262,67 & - & 277,05 \\
\hline Traumatismo intracraniano & $1.113,19$ & 816,87 & 767,94 & $1.179,99$ & - & - & $1.064,77$ & - & $1.000,44$ \\
\hline Traumatismo do tórax & 517,90 & - & - & 310,00 & - & - & 391,76 & - & 445,18 \\
\hline Traumatismo intra-abdominal & 755,69 & 428,69 & 770,90 & 877,71 & 507,67 & - & 909,61 & - & 813,78 \\
\hline Traumatismos múltiplos & 800,70 & 165,00 & 149,50 & 141,55 & - & - & 260,87 & - & 671,26 \\
\hline Queimaduras & - & - & 428,60 & - & 805,11 & - & - & - & 497,06 \\
\hline Intoxicações & - & - & 231,66 & - & 432,16 & 99,11 & $1.106,96$ & - & 473,60 \\
\hline Complicações de cuidados médicos & - & - & - & - & - & 334,27 & - & - & 334,27 \\
\hline Seqüelas & - & - & - & - & - & - & - & 501,33 & 501,33 \\
\hline Demais lesões & 456,08 & 194,76 & 179,58 & 192,68 & - & - & 180,11 & - & 233,52 \\
\hline Todas as lesões & 614,63 & 472,48 & 315,76 & 594,90 & 500,61 & 330,35 & 393,61 & 501,33 & 489,26 \\
\hline
\end{tabular}


Gasto médio de internação (em Reais) e razão de gasto médio óbito/alta por lesões e causas externas, segundo tipo de saída hospitalar. Hospital Municipal de São José dos Campos, São José dos Campos, São Paulo, Brasil, primeiro semestre de 2003.

\begin{tabular}{|c|c|c|c|}
\hline & \multicolumn{2}{|c|}{ Gasto médio de internação } & \multirow[t]{2}{*}{ Razão 2/1 } \\
\hline & Alta (1) & Óbito (2) & \\
\hline \multicolumn{4}{|l|}{ Segmento corporal afetado } \\
\hline Cabeça & 621,33 & $1.262,72$ & $2,0: 1$ \\
\hline Pescoço & 980,26 & - & - \\
\hline Tórax & 535,15 & $1.575,58$ & $2,9: 1$ \\
\hline Abdome, dorso e pelve & 629,73 & 938,67 & $1,5: 1$ \\
\hline Membros superiores & 231,99 & - & - \\
\hline Membros inferiores & 444,96 & 860,76 & $1,9: 1$ \\
\hline Traumatismos múltiplos & 470,52 & $2.477,95$ & $5,3: 1$ \\
\hline \multicolumn{4}{|l|}{ Natureza da lesão } \\
\hline Fraturas & 397,19 & 860,76 & $2,2: 1$ \\
\hline Crânio e ossos da face & 224,03 & - & - \\
\hline Pescoço & $1.191,42$ & - & - \\
\hline Tórax & 605,31 & - & - \\
\hline Coluna lombar e pelve & 648,97 & - & - \\
\hline Ombro e braço & 265,31 & - & - \\
\hline Antebraço & 273,15 & - & - \\
\hline Punho e mão & 167,06 & - & - \\
\hline Fêmur & 606,29 & 860,76 & $1,4: 1$ \\
\hline Perna & 368,49 & - & - \\
\hline Pé & 204,45 & - & - \\
\hline Luxações, entorses e distensões & 277,05 & - & - \\
\hline Traumatismo intracraniano & 949,01 & $1.262,72$ & $1,3: 1$ \\
\hline Traumatismo do tórax & 364,43 & $1.575,58$ & $4,3: 1$ \\
\hline Traumatismo intra-abdominal & 776,18 & $1.017,94$ & $1,3: 1$ \\
\hline Traumatismos múltiplos & 470,52 & $2.477,95$ & $5,3: 1$ \\
\hline Queimaduras & 497,06 & - & - \\
\hline Intoxicações & 473,60 & - & - \\
\hline Complicações de cuidados médicos & 334,27 & - & - \\
\hline Seqüelas & 501,33 & - & - \\
\hline Demais lesões & 282,28 & 383,77 & $1,4: 1$ \\
\hline \multicolumn{4}{|l|}{ Tipo de causa externa } \\
\hline Acidentes de transporte & 560,97 & $1.464,79$ & $2,6: 1$ \\
\hline Quedas & 437,61 & $1.193,19$ & $2,7: 1$ \\
\hline Demais causas acidentais & 315,76 & - & - \\
\hline Agressões & 585,30 & 702,90 & $1,2: 1$ \\
\hline Lesões autoprovocadas intencionalmente & 500,61 & - & - \\
\hline Complicações da assistência médica & 330,35 & - & - \\
\hline Causas indeterminadas & 368,63 & $1.517,99$ & $4,1: 1$ \\
\hline Seqüelas & 501,33 & - & - \\
\hline
\end{tabular}

têm maior custo-dia e menor tempo médio de permanência do que as internações por causas naturais 1. Em São José dos Campos, os gastos do SUS com internações por traumas são crescentes, já que houve um aumento do número de internações e de procedimentos realizados. Em 1998, os repasses de recursos do Ministério da Saúde foram de R $\$ 732.038,43$, enquanto em 2004, de R\$1.573.001,942. Os valores que o Ministério da Saúde pagou pelas internações hospitalares feitas pelo SUS referem-se à remuneração dos gastos diretos médico-hospitalares e estão defasados em relação aos custos reais efetivamente despendidos pelos hospitais. 
Em relação ao segmento corporal afetado, o gasto médio de internação em São José dos Campos foi maior para pescoço, cabeça, múltiplas regiões e abdome, em ordem decrescente. No Estado de São Paulo, em 2003, os segmentos corporais com maior gasto médio de internação por causas externas foram: pescoço (R\$1.433,29), quadril e coxa (R\$ 1.241,46), tórax ( $\mathrm{R} \$ 957,27)$ e abdome (R\$ 898,27) (Mello Jorge MHP, Koizumi MS. Eixo II - Avaliação dos Custos Diretos na Atenção à Saúde das Vítimas de Violência: Uma Análise das Internações Hospitalares. Relatório de pesquisa apresentado à Fundação de Amparo à Pesquisa do Estado de São Paulo; 2006).

$\mathrm{O}$ maior gasto médio de internação em pacientes internados com lesões decorrentes de agressões e acidentes de transporte, em relação às mesmas lesões por outras causas, pode significar que os maiores custos com o tratamento de alguns traumas foram conseqüência da maior gravidade das lesões nesses tipos de causas externas, o que acarretou a realização de procedimentos de maior complexidade.

Houve um predomínio das internações por acidentes de transporte tanto na proporção de internações como na proporção de gastos, com as quedas em segundo lugar em ambas as situações. Esse perfil encontrado diferiu muito daquele do Estado de São Paulo ${ }^{3}$. Em 2005, neste Estado, a proporção de internações por causas externas foi de $48 \%$ para as quedas e $17 \%$ para os acidentes de transporte, enquanto a proporção de gastos distribuiu-se em $41 \%$ para as quedas e $22 \%$ para acidentes de transporte.

A elevada proporção de internações e gastos por causas indeterminadas, encontrada no presente estudo, pode ser em explicada, em parte, pelo registro incompleto, no prontuário do paciente, de prováveis agressões.

No outro extremo dos gastos, ficaram as complicações da assistência médica, com menor gasto médio e custo-dia. Esse perfil deveu-se à natureza dessas lesões, que foram principalmente as infecções pós-cirúrgicas, cujo tratamento requer, na maioria das vezes, procedimentos médicos de recuperação mais rápida.

Mello Jorge \& Koizumi (Relatório de pesquisa; 2006) encontraram resultados semelhantes aos do presente estudo nas internações pelo SUS por causas externas no Estado de São Paulo, de 2000 a 2003, onde predominaram os acidentes de transporte e os homicídios como os tipos de causas externas com maior gasto médio de internação. No Rio de Janeiro, em 1995, foi realizado estudo minucioso de custos do atendimento de emergência por causas externas em dois hospitais públicos 15. Em um dos hospitais, os acidentes de transporte e as agressões foram responsáveis por, respectivamente, $74,3 \%$ e $24,9 \%$ dos custos; no outro, as agressões representaram $49,8 \%$ dos custos e os acidentes de transporte, $48,4 \%$. Apesar das diferenças de metodologia para obtenção dos dados, parece haver uma efetiva predominância dos gastos com acidentes de transportes e agressões no conjunto da morbidade mais grave, não fatal, das causas externas.

As quedas, segundo lugar nas internações por causas externas, no hospital municipal, representaram o quinto maior gasto médio de internação. No Estado de São Paulo, apesar de serem a primeira causa de internação, as quedas também tiveram seu gasto médio de internação menor do que os acidentes de transporte, agressões, seqüelas e demais causas acidentais (Mello Jorge MHP, Koizumi MS. Relatório de pesquisa; 2006).

Em relação ao diferencial de gasto médio de internação segundo o tipo de saída hospitalar, resultado semelhante foi encontrado para o Brasil, no ano 2000 1. No referido estudo, todas as internações pelo SUS por causas externas que evoluíram para óbito tiveram, pelo menos, o dobro do gasto médio do que as internações que tiveram saída por alta hospitalar. No Estado de São Paulo, em 2003, as internações por causas externas com maiores razões foram: suicídios e tentativas $(3,9: 1)$, ignoradas $(3,0: 1)$, demais acidentes $(2,5: 1)$, fatores suplementares $(2,5: 1)$, quedas $(2,6: 1)$, seqüelas $(2,3: 1)$, acidentes de transporte, homicídios e tentativas de homicídio (1,9:1 cada) (Mello Jorge MHP, Koizumi MS. Relatório de pesquisa; 2006).

Foi relevante, no presente estudo e no de Mello Jorge \& Koizumi (Relatório de pesquisa; 2006), o resultado de maior razão de gasto médio óbito/alta nas causas indeterminadas/ignoradas. Reitera-se aqui que agravos de importante gravidade deixaram de ser devidamente classificados em relação a sua freqüência e gastos, comprometendo a qualidade da análise.

Uma explicação para o fato de as internações por acidentes de transporte e por agressões não terem tido um acréscimo tão importante no seu custo em relação às demais causas pode ser o seu maior gasto médio, decorrente de lesões mais graves, mesmo nas internações sem evolução para óbito. O aumento de custo nos casos mais graves acaba por influenciar mais as internações de menor gasto médio. Esse fenômeno foi particularmente evidenciado por Mello Jorge \& Koizumi 1, que identificaram maiores acréscimos de gastos quando ocorria evolução para óbito nas internações por envenenamento, penetração de corpo estranho e demais acidentes.

O padrão de gastos governamentais com internações por causas externas, neste estudo, foi semelhante ao encontrado em outros estu- 
dos realizados com base no banco de dados do SIH-SUS. As principais diferenças foram relacionadas a algumas características específicas, como o fato de o presente estudo ter sido realizado em um único hospital e também de ter sido realizada uma verificação da qualidade da informação nos prontuários dos pacientes.

A proporção dos gastos segundo tipo de causa externa mostrou um perfil muito diferente em relação ao Estado de São Paulo. No hospital municipal de São José dos Campos, houve um predomínio das internações por acidentes de transporte em relação às quedas, tanto na freqüência como nos gastos, o que permite concluir que os acidentes de transporte representam um problema importante para o custeio da assistência hospitalar pública no município.

Uma explicação para a diferença encontrada na distribuição proporcional das internações por acidentes de transporte e quedas em relação aos demais estudos seria a má qualidade da codificação do diagnóstico de causas externas, na maioria dos municípios brasileiros, principalmente "quedas". Em Londrina, Estado do Paraná, em 2004, Tomimatsu 16 identificou uma mudança significativa na proporção de internações por quedas no SIH-SUS, após correção dos dados, de $56,7 \%$ para $16,4 \%$.

As agressões também significaram altos custos de internação, não como gastos totais, mas como gasto médio e custo-dia. Isso se deve à maior gravidade das lesões. A freqüência baixa das internações por agressões, em relação ao número de óbitos pela mesma causa, seria explicada pela sua alta letalidade no local de ocorrência e pelo sub-registro no hospital, seja por receio do paciente em revelar a agressão, seja por desinteresse/receio dos profissionais de saúde em coletar e registrar tal informação.
As internações por acidentes de transporte e agressões tiveram os maiores gastos médico-hospitalares, provavelmente por causa da ocorrência de lesões mais graves, com realização de procedimentos de maior complexidade nesses tipos de agravos e/ou maior tempo de permanência no hospital. O gasto médio de internação para os pacientes que faleceram foi maior do que para os pacientes que saíram por alta, em todos os agrupamentos de natureza da lesão em que ocorreu óbito, provavelmente pelas mesmas razões.

O fato de o atendimento médico-hospitalar para pacientes internados por lesões decorrentes de causas externas ser mais oneroso do que aquele relativo às causas naturais faz pensar, de imediato, na possibilidade de investimento em prevenção, a fim de diminuir a demanda aos hospitais, reduzindo, conseqüentemente, o sofrimento das vítimas e dos custos da assistência médica. Este trabalho mostrou a importância dos acidentes de transporte nesses gastos e a maior possibilidade de preveni-los (relativamente às agressões e lesões autoprovocadas intecionalmente), o que permite concluir que é nesse grupo de causas que as medidas preventivas devem ter seu início.

A principal motivação para a realização de estudos desse tipo no nível municipal é a possibilidade de alertar os gestores públicos e a sociedade sobre a realidade local. Ao se confirmarem ou refutarem os resultados de estudos mais amplos existentes na literatura, é possível desencadear discussões mais efetivas para a elaboração de políticas públicas voltadas para o problema.

O valor educativo da divulgação desses resultados para a população mais jovem, ainda não exposta com freqüência aos riscos identificados, pode auxiliar na prevenção da ocorrência desses agravos.

\section{Resumo}

O objetivo do estudo foi mensurar os gastos diretos do Sistema Único de Saúde (SUS) com internações por causas externas em São José dos Campos, São Paulo, Brasil. Foram estudadas as internações por lesões decorrentes de causas externas, respectivamente capítulos XIX e XX da CID-10, no primeiro semestre de 2003, no Hospital Municipal Dr. José de Carvalho Florence. Foram analisados os valores pagos através do SUS, após a verificação da qualidade dos dados nos prontuários de 976 internações. Os maiores gastos totais foram por internações decorrentes de acidentes de transporte e quedas. O maior gasto médio de internação foi por acidentes de transporte $(R \$ 614,63)$, seguido das agressões $(R \$ 594,90)$. As lesões que representaram maior gasto médio foram as fraturas de pescoço ( $R \$$ $1.191,42)$ e traumatismo intracraniano $(R \$ 1.000,44)$. As internações com maior custo-dia foram fraturas do crânio e dos ossos da face $(R \$ 166,72)$ e traumatismo intra-abdominal $(R \$ 148,26)$. Os resultados encontrados demonstraram que os acidentes de transporte, as quedas e as agressões são importantes fontes de gastos com internações por causas externas no município.

Gastos em Saúde; Causas Externas; Hospitalização; Sistema Único de Saúde 


\section{Colaboradores}

L. P. R. Melione foi responsável pela concepção e desenho do artigo, análise dos bancos de dados, interpretação dos resultados, levantamento bibliográfico e elaboração do texto. M. H. P. Mello-Jorge colaborou no desenho do artigo, na discussão dos resultados, no levantamento bibliográfico, bem como na revisão e correções do conteúdo do artigo.

\section{Referências}

1. Mello Jorge MHP, Koizumi MS. Gastos governamentais do SUS com internações hospitalares por causas externas: análise no Estado de São Paulo, 2000. Rev Bras Epidemiol 2004; 7:228-38.

2. Departamento de Informática do SUS. Movimento mensal de internação hospitalar. http://msbbs. datasus.gov.br/public/default.htm (acessado em 03/Abr/2006).

3. Grupo Técnico de Prevenção de Acidentes e Violências, Centro de Vigilância Epidemiológica "Prof. Alexandre Vranjac", Coordenadoria de Controle de Doenças, Secretaria de Estado da Saúde de São Paulo. O impacto dos acidentes e violências nos gastos da saúde. Rev Saúde Pública 2006; 40:553-6.

4. Bittencourt SA, Camacho LAB, Leal MC. O Sistema de Informação Hospitalar e sua aplicação na saúde coletiva. Cad Saúde Pública 2006; 22:19-30.

5. Hendrie D, Hall SE, Arena G, Legge M. Health system costs of falls of older adults in Western Australia. Aust Health Rev 2004; 28:363-73.

6. Instituto de Pesquisa Econômica Aplicada. Impactos sociais e econômicos dos acidentes de trânsito nas aglomerações urbanas. Brasília: Instituto de Pesquisa Econômica Aplicada; 2003.

7. Dueck A, Poenaru D, Pichora DR. Cost factors in Canadian pediatric trauma. Can J Surg 2001; 44:117-21.

8. Iunes RF. III - Impacto econômico das causas externas no Brasil: um esforço de mensuração. Rev Saúde Pública 1997; 31(4 Suppl):38-46.

9. Ministério da Saúde. Tabela de procedimentos do SIA/SIH-SUS. http://dtr2004.saude.gov.br/sas/ links.php (acessado em 07/Jul/2006).
10. Ministério da Saúde. Portaria nº. 142, de 13 de novembro de 1997. Dispõe sobre o preenchimento de Autorização de Internação Hospitalar - AIH, em casos com quadro compatível com causas externas. Diário Oficial da União 1997; 17 nov.

11. Organização Mundial da Saúde. Classificação estatística internacional de doenças e problemas relacionados à saúde, 10a revisão. São Paulo: Centro Colaborador da OMS para a Classificação de Doenças em Português; 1995.

12. Prefeitura Municipal de São José dos Campos. São José em dados, 2004: informações sobre a cidade de São José dos Campos. São José dos Campos: Prefeitura Municipal de São José dos Campos; 2004.

13. Moreira ML. Cobertura e utilização de serviços de saúde suplementar no Estado de São Paulo [Dissertação de Mestrado]. Rio de Janeiro: Escola Nacional de Saúde Pública, Fundação Oswaldo Cruz; 2004.

14. Melione LPR. Morbidade hospitalar por causas externas no Sistema Único de Saúde em São José dos Campos, SP [Dissertação de Mestrado]. São Paulo: Faculdade de Saúde Pública, Universidade de São Paulo; 2006.

15. Deslandes SF, Silva CMFP, Ugá MAD. O custo do atendimento emergencial às vítimas de violências em dois hospitais do Rio de Janeiro. Cad Saúde Pública 1998; 14:287-99.

16. Tomimatsu MFAI. Internações por acidentes e violências financiadas pelo setor público em Londrina, Paraná: análise dos registros, gastos e causas [Dissertação de Mestrado]. Londrina: Universidade Estadual de Londrina; 2006.

Recebido em 06/Jun/2007

Versão final reapresentada em 19/Dez/2007

Aprovado em 26/Dez/2007 